\title{
Utility of concomitant tricuspid valve procedures for patients undergoing implantation of a continuous-flow left ventricular device
}

\author{
Valentino Piacentino III, MD, PhD, ${ }^{\mathrm{a}}$ Asvin M. Ganapathi, MD, ${ }^{\mathrm{a}}$ Mark Stafford-Smith, MD, \\ Michael K. Hsieh, BS, ${ }^{a}$ Chetan B. Patel, MD,${ }^{\mathrm{c}}$ Alan A. Simeone, MD, ${ }^{\mathrm{a}}$ Joseph G. Rogers, MD, ${ }^{\mathrm{c}}$ and \\ Carmelo A. Milano, MD $^{\mathrm{a}}$
}

\begin{abstract}
Objective: Patients referred for implantable continuous-flow left ventricular assist devices (cfLVAD) frequently have preoperative right heart failure and tricuspid regurgitation (TR). The objective of this report is to examine early clinical benefits of concomitant tricuspid surgery for these patients.
\end{abstract}

\begin{abstract}
Methods: Sixty-one of 200 consecutive cfLVAD patients at our institution displayed preimplant right heart dysfunction and significant TR. Thirty-three underwent cfLVAD plus a tricuspid valve procedure (TVP), and 28 had cfLVAD alone. Preimplant characteristics and clinical outcomes were retrospectively studied. As previously described, post-LVAD right ventricular failure was defined as need for right ventricular assist device (RVAD) support or greater than 14 days of intravenous inotropic support.

Results: Preimplant characteristics were similar between the 2 groups. Cardiopulmonary bypass time was increased for the group that received concomitant TVPs. The most common TVP consisted of an undersizing ring annuloplasty. The cfLVAD-alone group had greater TR after implant relative to the cfLVAD+TVP group. The cfLVAD-alone group experienced greater postprocedure right ventricular failure relative to cfLVAD+TVP $(46.4 \%$ vs $18.2 \% ; P<.05)$. Furthermore, prolonged hospitalization was increased for the cfLVAD-alone group versus the cfLVAD+TVP. Survival was similar between the 2 groups.
\end{abstract}

Conclusions: Concomitant TVP appears to reduce postprocedure right ventricular failure for patients with significant TR undergoing cfLVAD implantation. (J Thorac Cardiovasc Surg 2012;144:1217-21)

Patients referred for support with an implantable left ventricular assist device (LVAD) frequently have right ventricular (RV) failure in addition to left heart failure. Some of these patients continue to manifest RV failure after LVAD implantation, which results in the need for RV assist device (RVAD) support or prolonged intravenous inotropic agent support. The advent of continuous-flow designs for LVAD pumps has resulted in reduced post-LVAD RV failure, but this complication continues to affect more than $20 \%$ of patients in recent LVAD trials. ${ }^{1}$ Furthermore, the presence of post-LVAD RV failure, even in patients with continuousflow LVADs (cfLVADs), is associated with reduced survival. ${ }^{2}$ This complication may also affect duration of hospitalization, quality of life, and functional status for cfLVAD recipients.

Most patients with RV failure who undergo implantable LVAD surgery also have significant tricuspid valve

\footnotetext{
From the Divisions of Cardiac and Thoracic Surgery, ${ }^{a}$ Cardiothoracic Anesthesia, ${ }^{b}$ and Cardiology, ${ }^{\mathrm{c}}$ Duke University Medical Center, Durham, NC.

Disclosures: Authors have nothing to disclose with regard to commercial support. V.P. and A.M.G. contributed equally to this investigation.

Read at the 92nd Annual Meeting of The American Association for Thoracic Surgery, San Francisco, California, April 28-May 2, 2012.

Received for publication May 4, 2012; revisions received July 2, 2012; accepted for publication July 26, 2012; available ahead of print Aug 31, 2012

Address for reprints: Carmelo A. Milano, MD, Duke University Medical Center, Box 3043, Durham, NC 27710 (E-mail: milan002@mc.duke.edu). $0022-5223 / \$ 36.00$

Copyright (c) 2012 by The American Association for Thoracic Surgery http://dx.doi.org/10.1016/j.jtcvs.2012.07.064
}

regurgitation (TR). ${ }^{3}$ Usually this $\mathrm{TR}$ is functional and reflects RV pressure or volume overload. The benefits of a concomitant tricuspid valve procedure (TVP) to correct TR at the time of LVAD implantation have not been rigorously examined. We previously reviewed the impact of concomitant tricuspid valve surgery in a cohort of patients that included many who received first-generation pulsatile LVADs. ${ }^{4}$ In this study, the role of concomitant tricuspid valve surgery is examined in a more modern experience consisting solely of patients having a cfLVAD.

In this study, a consecutive group of patients undergoing cfLVAD implantation with RV failure and significant TR are examined. The benefits of the concomitant TVP are studied as some of the patients received a concomitant TVP and others did not. This study focuses specifically on whether concomitant TVP is associated with reduced RV failure after the LVAD implantation. These results should provide insight for the clinical judgment of when concomitant tricuspid surgery should be attempted. Furthermore, it examines whether concomitant tricuspid surgery may be a strategy to avoid the need for mechanical RV support.

\section{METHODS}

Permission for this study was granted by the Institutional Review Board of Duke University Medical Center, and need for patient consent was waived. From 2005 to 2011, 200 consecutive cfLVADs were implanted at a single institution (Duke). Of the 200 patients, 61 had significant preimplant TR on preoperative echocardiography; these patients 


$$
\begin{aligned}
& \text { Abbreviations and Acronyms } \\
& \text { cfLVAD }=\text { continuous-flow left ventricular assist } \\
& \text { device } \\
& \text { CPB }=\text { cardiopulmonary bypass } \\
& \text { LVAD }=\text { left ventricular assist device } \\
& \text { RV }=\text { right ventricular } \\
& \text { RVAD }=\text { right ventricular assist device } \\
& \text { TR }=\text { tricuspid regurgitation } \\
& \text { TVP }=\text { tricuspid valve procedure }
\end{aligned}
$$

of patients who underwent implantation during the same era, with similar surgeons, intensive care unit staffing, and treatment protocols. Tricuspid annular dimensions were similar between the cfLVAD-alone group and the group that received the concomitant TVPs. The total CPB time was increased for the group receiving the concomitant TVPs by approximately 1 hour.

Table 2 shows the type of TVPs for the cfLVAD and TVP groups. A minority of patients required replacement with a bioprosthesis; there were no mechanical replacements. The most common procedure consisted of an undersizing annuloplasty ring repair. We have recently begun using semirigid rings for tricuspid annuloplasty.

Figure 1 depicts echocardiographic TR grade before and after LVAD implantation in the cfLVAD-alone and cfLVAD+TVP groups. Almost 50\% of the patients in the cfLVAD-alone group continued to demonstrate significant (moderate or severe) TR after LVAD implantation. This is similar to our earlier study on TR in LVAD patients receiving pulsatile devices. ${ }^{4}$ The majority of patients in the cfLVAD+TVP group had insignificant TR (less than moderate) at follow-up echocardiogram.

Table 3 describes post-LVAD outcomes between the 2 groups. Patients in the cfLVAD-alone group were more likely to experience postoperative RV failure $(P<.05)$ and also more likely to require a postoperative RVAD $(P<.05)$. Postimplant inotropic agent use also tended to be greater in the cfLVAD-alone group versus the cfLVAD+TVP group (12.1 vs 9.72 days; $P=.16$ ). Postimplant acute kidney injury tended to be less in the cfLVAD+TVP group versus the cfLVAD-alone group. Also, the cfLVAD-alone group had statistically more patients requiring prolonged hospitalization $(>30$ days; $P<.05)$ when compared with the cfLVAD+TVP group. Mortality rates were similar for the 2 groups.

$\mathrm{RV}$ failure is composed of the fraction of patients requiring postoperative RVADs or prolonged intravenous inotropic support. Figure 2 illustrates the incidence of RV failure in both groups after LVAD implantation. Forty-six percent of patients in the cfLVAD-alone group exhibited post-LVAD RV failure versus only $18 \%$ of the cfLVAD+TVP group; most of the difference resulted from greater RVAD requirement for the cfLVAD-alone group. The overall incidence of RV failure for the cfLVAD-alone group is greater than that of previous series, which is consistent with the fact that these patients represent a high risk group for RV failure. ${ }^{2}$ This statement is supported by data from Table 1, which showed reduced RV systolic volume index and increased ratio of central venous pressure to pulmonary capillary wedge pressure for both groups.

\section{DISCUSSION}

Recent studies have suggested that use of cfLVADs versus older pulsatile LVADs is associated with a reduced 
TABLE 1. Pre-cfLVAD patient characteristics

\begin{tabular}{|c|c|c|c|}
\hline & $\begin{array}{l}\text { cfLVAD-alone } \\
\quad(\mathbf{n}=\mathbf{2 8})\end{array}$ & $\begin{array}{l}\text { cfLVAD+TVP } \\
\quad(\mathbf{n}=\mathbf{3 3})\end{array}$ & $\begin{array}{c}P \\
\text { value }\end{array}$ \\
\hline Age $(y)$ & 59 & 56 & .15 \\
\hline Sex ( $\%$ male $)$ & 54 & 66 & .45 \\
\hline Heart failure etiology $(\%$ NICM $)$ & 64 & 44 & .12 \\
\hline BUN & 32 & 40 & .08 \\
\hline Creatinine (mg/dL) & 1.7 & 1.6 & .46 \\
\hline Heart rate (beats/min) & 93 & 88 & .27 \\
\hline CVP (mm Hg) & 18 & 19 & .16 \\
\hline Mean PAP (mm Hg) & 38 & 38 & .27 \\
\hline PCWP (mm Hg) & 27 & 26 & .44 \\
\hline $\begin{array}{l}\text { Pulmonary vascular resistance } \\
\text { (Wood inits) }\end{array}$ & 3.6 & 3.4 & .30 \\
\hline CVP/PCWP & 0.651 & 0.739 & .10 \\
\hline Cardiac index $\left(\mathrm{L} / \mathrm{min} / \mathrm{m}^{2}\right)$ & 1.8 & 1.78 & .45 \\
\hline RVSWI (mL/min/m²) & 410 & 446 & .32 \\
\hline $\begin{array}{l}\text { Mixed venous oxygen } \\
\text { saturation }(\%)\end{array}$ & 46 & 46 & .37 \\
\hline Preoperative ventilator (\%) & 14 & 13 & 1.0 \\
\hline Year of most implantations & 2008 & 2010 & \\
\hline Era of implantations & 2005-2011 & 2005-2011 & \\
\hline CPB times (min) & 111 & 179 & $<.001$ \\
\hline $\begin{array}{l}\text { Nontricuspid concomitant } \\
\text { procedures }(\%)\end{array}$ & 19 & 18 & .4 \\
\hline $\begin{array}{l}\text { Preimplant TV annular diameter } \\
\text { (4-chamber view) }(\mathrm{cm})\end{array}$ & 4.35 & 4.38 & NS \\
\hline $\begin{array}{l}\text { Preimplant TV annular diameter } \\
(\mathrm{RV} \text { inflow and outflow view) } \\
(\mathrm{cm})\end{array}$ & 4.66 & 4.58 & NS \\
\hline \multicolumn{4}{|c|}{$\begin{array}{l}c f L V A D \text {-alone, Continuous-flow left ventricular assist device alone; } c f L V A D+T V P \text {, } \\
\text { continuous-flow left ventricular assist device plus tricuspid valve procedure; } \\
N I C M \text {, nonischemic cardiomyopathy; } B U N \text {, blood urea nitrogen; } C V P \text {, central venous } \\
\text { pressure; } P A P \text {, pulmonary artery pressure; } P C W P \text {, pulmonary capillary wedge pres- } \\
\text { sure; } R V S W I \text {, Right Ventricular Stroke Work Index; } C P B \text {, cardiopulmonary bypass; } \\
T V \text {, tricuspid valve; } R V \text {, right ventricular; } N S \text {, not significant. }\end{array}$} \\
\hline
\end{tabular}

incidence of RV failure. ${ }^{1}$ Nevertheless, even in an era of cfLVADs, RV failure remains an important postimplant complication that affects $20 \%$ of patients and negatively affects survival outcomes. ${ }^{1,2}$ While preimplant risk factors for this complication have been described, effective treatment or preventive measures are not well understood. ${ }^{1,2} \mathrm{We}^{3}$ previously reported that TR is commonly encountered before LVAD implantation, does not resolve with mechanical left ventricular unloading, and is associated with postimplant $\mathrm{RV}$ failure. In this report, in a cohort of patients with

TABLE 2. Type of tricuspid valve procedures

\begin{tabular}{lr}
\hline \multicolumn{1}{c}{ Procedure } & No. \\
\hline Bioprosthetic replacement & 4 \\
Undersizing ring annuloplasty repair & 29 \\
Flexible ring & 22 \\
Semirigid ring & 7 \\
Total & 33 \\
\hline
\end{tabular}

significant TR who received cfLVADs, we examine the benefits of concomitant TVPs to reduce postimplant RV failure.

Recently, Maltais and associates ${ }^{5}$ reported the echocardiographic changes associated with cfLVAD support and concomitant tricuspid valve annuloplasty. Repeat echocardiography at 1 month after implant demonstrated that the group that received concomitant TVP displayed reduced TR and decreased RV volume. The current report compliments this echocardiographic study by examining the clinical outcomes that define RV failure for a cohort of cfLVAD patients with significant TR who undergo either concomitant tricuspid valve surgery or LVAD implantation alone. Importantly, postimplant RV failure, defined by need for RVAD support or prolonged intravenous inotropic infusion ( $>14$ days), is reduced in the group undergoing concomitant TVPs. This study suggests that concomitant tricuspid surgery may be a simple intraoperative strategy to help avoid RV failure.

Our earlier study of TVP during pulsatile or nonpulsatile LVAD implantation suggested a survival benefit for concomitant TVP, and a recent study from Atrache and colleagues ${ }^{6}$ also suggests a survival benefit of concomitant TVP and LVAD implantation. ${ }^{4}$ The current report and that of Maltais and coworkers ${ }^{5}$ do not demonstrate a survival benefit associated with concomitant TVPs. Several possible explanations can be offered for the equivalent survival outcomes found in this report and that of Maltais' group. ${ }^{5}$ First, this report examines a relatively small number of subjects and may be inadequately powered to show survival differences. Furthermore, this study does not randomize patients to receive concomitant TVPs, and the group that received the TVPs may have had worse RV performance before the LVAD implantation relative to those who were delegated to not undergo the concomitant procedure. For example, the group that underwent the concomitant TVPs shows a trend to have higher preoperative central venous pressure/pulmonary capillary wedge pressure ratios. The fact that patients were not randomized to either receive or not receive TVP is the single most important limitation to all conclusions. Also, a small fraction of the LVAD-alone group experienced improvement in their postimplant TR (even though they did not receive a procedure), which may have positively affected survival. Another explanation for the equivalent survival for the 2 groups is that the group undergoing LVAD alone may have received a variety of additional postimplant treatments for RV failure (eg, pulmonary vasodilators) that allowed for better hemodynamics and survival.

Although this study supports the use of concomitant TVPs for LVAD patients with significant TR, several important questions remain. First, the group undergoing concomitant TVPs included both moderate and severe preimplant grades of TR. It is unclear what preoperative 

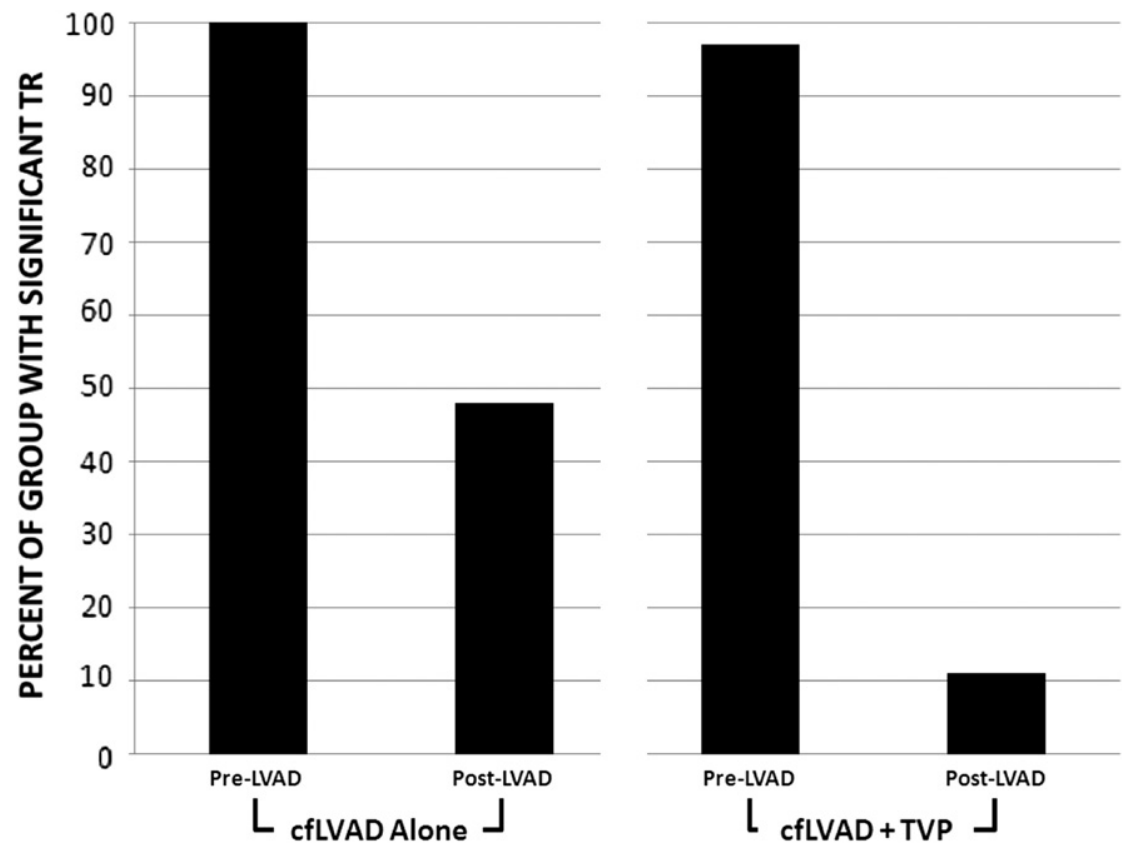

FIGURE 1. Preoperative echocardiographic tricuspid regurgitation $(T R)$ grade versus follow-up TR grade in cfLVAD-alone versus cfLVAD+TVP groups. cfLVAD-alone, Continuous-flow left ventricular assist device alone; $c L V A D+T V P$, continuous-flow left ventricular assist device plus tricuspid valve procedure; $L V A D$, left ventricular assist device.

TR severity would warrant annuloplasty. Furthermore, Dreyfus and colleagues ${ }^{7}$ suggest that the decision for annuloplasty in the setting of functional TR should be based on annular size (dilation) rather than severity of regurgitation. This line of thought suggests that the surgical annuloplasty may have a positive impact on RV reverse remodeling independent of reducing the volume loading associated with TR. Therefore, it is unclear whether tricuspid annuloplasty should be deployed for annular dilation alone. Another

TABLE 3. Clinical outcome measures post-cfLVAD implantation

\begin{tabular}{lccc}
\hline & cfLVAD-alone & cfLVAD+TVP & $\boldsymbol{P}$ value \\
\hline RV failure & $13 / 28(46.4 \%)$ & $6 / 33(18.2 \%)$ & $<.05$ \\
$\begin{array}{l}\text { Patients requiring }>30 \\
\quad 12 / 28(42.9 \%)\end{array}$ & $6 / 33(18.2 \%)$ & $<.05$ \\
$\quad \begin{array}{l}\text { days after LVAD } \\
\text { hospitalization }\end{array}$ & & & \\
$\begin{array}{l}\text { Post-cfLVAD acute } \\
\text { kidney injury }\end{array}$ & $10 / 28(32.14 \%)$ & $7 / 33(15.15 \%)$ & .21 \\
$\begin{array}{l}\text { Duration of } \\
\text { hospitalization }(\mathrm{d}) \\
\quad \text { average, median, }\end{array}$ & $32(20 ; 15,37)$ & $24(19 ; 13,27)$ & .21 \\
$\quad \begin{array}{l}\text { 25th, and 75th } \\
\text { percentiles) }\end{array}$ & & & \\
$\begin{array}{l}\text { Need for } \\
\text { rehospitalization in } \\
\text { first year }\end{array}$ & $17 / 19(89.47 \%)$ & $22 / 26(84.6 \%)$ & 1.0 \\
$\begin{array}{l}\text { Thirty-day mortality } \\
\text { One-year mortality }\end{array}$ & $1 / 28(3.5 \%)$ & $1 / 32(3.13 \%)$ & 1.0 \\
\hline f & $5 / 22(22.73 \%)$ & $3 / 24(12.5 \%)$ & .45 \\
\hline
\end{tabular}

cfLVAD-alone, Continuous-flow left ventricular assist device alone; cfLVAD $+T V P$, continuous-flow left ventricular assist device plus tricuspid valve procedure; important question relates to the deployment of RVAD support in patients who undergo cfLVAD implantation. This study suggests that many patients who may be considered for RVAD support may benefit from tricuspid annuloplasty and that this strategy should be attempted first before committing the patient to biventricular support, which

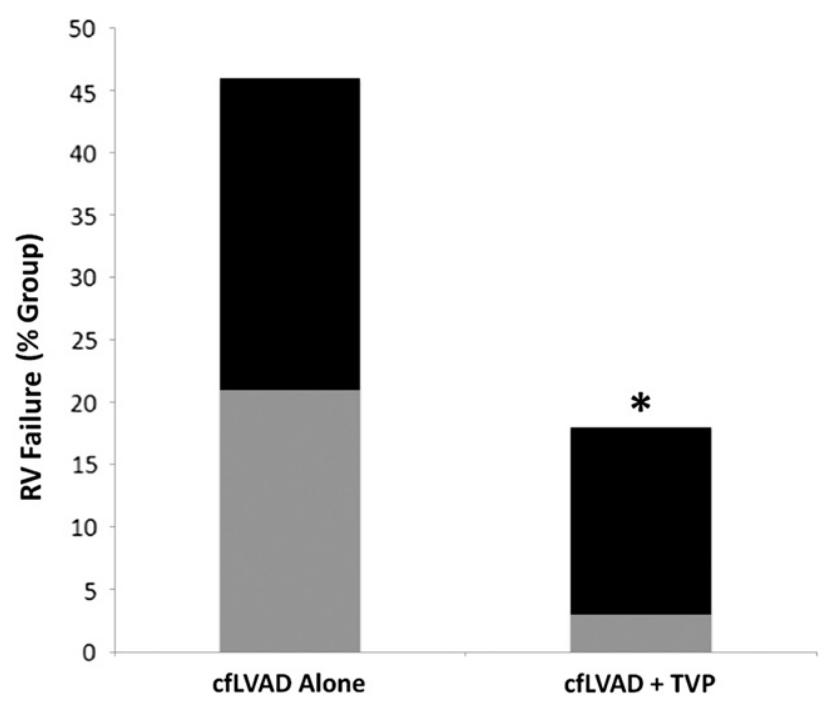

FIGURE 2. Postoperative right ventricular $(R V)$ failure for cfLVAD-alone versus cfLVAD+TVP. Bars represent the incidence of need for a right ventricular assist device ( gray) or prolonged inotrope use ( $>14$ days; black) for each group. $* P<.05$. cfLVAD-alone, Continuous-flow left ventricular assist device alone; $c f L V A D+T V P$, continuous-flow left ventricular assist device plus tricuspid valve procedure. 
adds to the complexity and cost of the mechanical circulatory support. In addition, tricuspid annuloplasty may be complementary to temporary RVAD support and may facilitate later weaning from the RVAD.

\section{CONCLUSIONS}

This study examines a modern cohort of patients undergoing implantation of a cfLVAD who displayed preoperative RV failure and significant TR. Roughly half of the group underwent LVAD alone, whereas the other half had concomitant TVPs. The group that underwent concomitant TVP required more prolonged CPB times but, despite this, experienced reduced postimplant RV failure as described by less need for RVAD support and reduced inotropic infusion support. Furthermore, prolonged hospitalizations were less frequent for the group that underwent concomitant TVP. Therefore, patients undergoing implantable cfLVADs who have moderate or severe TR should be considered for concomitant TVP to reduce postimplant RV dysfunction.

\section{References}

1. Slaughter MS, Rogers JG, Milano CA, Russell SD, Conte JV, Feldman D, et al, for the HeartMate II Investigators. Advanced heart failure treated with continuousflow left ventricular assist device. N Engl J Med. 2009;361:2241-51.

2. Kormos RL, Teuteberg JJ, Pagani FD, Russell SD, John R, Miller LW, et al. Right ventricular failure in patients with the HeartMate II continuous flow left ventricular assist device: incidence, risk factors, and impact on outcomes. J Thorac Cardiovasc Surg. 2010;139:1316-24.

3. Piacentino V 3rd, Williams ML, Depp T, Garcia-Huerta K, Blue L, Lodge AJ, et al. Impact of tricuspid valve regurgitation in patients treated with implantable left ventricular assist devices. Ann Thorac Surg. 2011;91:1342-7.

4. Piacentino V 3rd, Troupes CD, Ganapathi AM, Blue LJ, Mackensen GB, Swaminathan M, et al. Clinical impact of concomitant tricuspid valve procedures during left ventricular assist device implantation. Ann Thorac Surg. 2011;92: 1414-8.

5. Maltais S, Topilsky Y, Tchantchaleishvili V, McKellar SH, Durham LA, Joyce LD, et al. Surgical treatment of tricuspid valve insufficiency promotes early reverse remodeling in patients with axial-flow left ventricular assist devices. J Thorac Cardiovasc Surg. 2012;143:1370-6.

6. Atrache ME, Brewer R, Nemeh H, Williams C, Tita C, Paone G, et al. Tricuspid repair at the time of LVAD implantation is associated with improved survival. JAm Coll Cardiol. 2012;59:881.

7. Dreyfus GD, Corbi PJ, Chan KMJ, Bahrami T. Secondary tricuspid regurgitation or dilatation: which should be the criteria for surgical repair? Ann Thorac Surg. 2005;79:127-32. 